Bismuth-Loaded Polymer Scintillators for Gamma Ray Spectroscopy

B. L. Rupert, N. J. Cherepy, B. W. Sturm, R. D. Sanner, Z. Dai, S. A. Payne

April 12, 2011

Materials Research Society Spring Meeting San Francisco, CA, United States April 25, 2011 through April 29, 2011 
This document was prepared as an account of work sponsored by an agency of the United States government. Neither the United States government nor Lawrence Livermore National Security, LLC, nor any of their employees makes any warranty, expressed or implied, or assumes any legal liability or responsibility for the accuracy, completeness, or usefulness of any information, apparatus, product, or process disclosed, or represents that its use would not infringe privately owned rights. Reference herein to any specific commercial product, process, or service by trade name, trademark, manufacturer, or otherwise does not necessarily constitute or imply its endorsement, recommendation, or favoring by the United States government or Lawrence Livermore National Security, LLC. The views and opinions of authors expressed herein do not necessarily state or reflect those of the United States government or Lawrence Livermore National Security, LLC, and shall not be used for advertising or product endorsement purposes. 


\title{
Bismuth-Loaded Polymer Scintillators for Gamma Ray Spectroscopy
}

Benjmain L. Rupert, Nerine J. Cherepy, Benjamin W. Sturm, Robert D. Sanner, Zurong Dai and Stephen A. Payne

Lawrence Livermore National Laboratory, Livermore, CA 94550, USA

\begin{abstract}
We synthesize a series of polyvinylcarbazole monoliths containing varying loadings of triphenyl bismuth as a high- $Z$ dopant and varying fluors, either organic or organometallic, in order to study their use as scintillators capable of gamma ray spectroscopy. A trend of increasing bismuth loading resulting in a better-resolved photopeak is observed. For PVK parts with no fluor or a standard organic fluor, diphenylanthracene, increasing bismuth loading results in decreasing light yield while with samples 1 or $3 \%$ by weight of the spin-orbit coupling organometallic fluor FIrpic, which emits light from both singlet and triple excitons, show increasing light yield with increasing bismuth loading. Our best performing $\mathrm{PVK} / \mathrm{BiPh}_{3} / \mathrm{FIrpic}$ scintillator with $40 \mathrm{wt} \% \mathrm{BiPh}_{3}$ and $3 \mathrm{wt} \%$ FIrpic has an emission maximum of $500 \mathrm{~nm}$, a light yield of $\sim 30,000$ photons/MeV, and energy resolution better than 7\% FWHM at $662 \mathrm{keV}$. Replacing the Ir complex with an equal weight of diphenylanthracene produces a sample with a light yield of $\sim 6,000$ photons/MeV, with an emission maximum at $420 \mathrm{~nm}$ and energy resolution of $9 \%$ at $662 \mathrm{keV}$. Transmission electron microscopy studies show that the $\mathrm{BiPh}_{3}$ forms small clusters of approximately $5 \mathrm{~nm}$ diameter.
\end{abstract}

\section{INTRODUCTION}

Due to their low cost and ease of fabrication in large volumes, plastic scintillators find many uses in radiation detection.. Scintillator-based gamma ray spectroscopy requires scintillator materials with high light yield, excellent light yield proportionality, prompt emission decay and high effective atomic number for high photoelectric cross-section. Unfortunately plastic scintillators have low light yields, and more importantly, their low effective $Z\left(Z_{\text {eff }} \approx 4.5\right)$ results in poor photopeak efficiency, and they therefore have not been used for gamma-ray spectroscopy. Attempts to increase $\mathrm{Z}_{\mathrm{eff}}$ by doping with heavy metals to induce a photopeak have been made in the past. ${ }^{1-3}$ However, difficulties due to poor solubility of the high- $Z$ component and quenching of luminescence were never resolved.

While the low light yields of polymer scintillators impacts their use for event counting to some extent, it becomes critical for gamma ray spectroscopy. Separate from any consideration of spectroscopy, Campbell and Crone produced thin films of polyvinylcarbazole (PVK) doped with an iridium complex fluor which were found to offer improved scintillation light yields. ${ }^{4}$ This is due to the same mechanism now widely employed in organic light emitting diodes (OLEDs), wherein it has been found that the Ir-complexes are capable of capturing and emitting both singlet and triplet excitons, due to spin-orbit coupling. While work with thin films demonstrated that higher light yields are possible with Ir-complexes, large scintillator volumes are needed for practical counting rates. Furthermore, if a high- $Z$ component can be introduced, without decreasing the light yield, the capabilities of such a material could go beyond the usual uses for plastic scintillator for gross counting, and be used as a gamma ray spectroscopy scintillator for radioisotope identification applications. 


\section{EXPERIMENT}

All materials were used as received, except N-vinyl carbazole (NVK), which was purified by vacuum sublimation. In a glass ampoule were combined $10 \mathrm{mg}$ Luperox 231, fluorescent or phosphorescent fluor as indicated in Table $1, \mathrm{BiPh}_{3}$ as indicated in Table 1 and enough $\mathrm{NVK}$ to bring the total mass to $1.0 \mathrm{~g}$. The ampoule was then placed under vacuum and refilled with nitrogen three times to remove oxygen from the system. While under a slight positive pressure of nitrogen the ampoule was placed in well in a fitted well in an aluminum heating block at $80^{\circ}$ $\mathrm{C}$ for 24 hours. After cooling again to room temperature the ampoules are broken and the scintillator parts shaped and polished to give right cylinders $18 \mathrm{~mm}$ in diameter and $2 \mathrm{~mm}$ in height.

Transmission Electron Microscopy (TEM) characterization was demonstrated under a Philips CM300-FEG instrument, operating at $300 \mathrm{kV}$. The instrument is a fully digital controlled system and has powerful and versatile capabilities including X-ray energy dispersive spectroscopy (EDS) and electron energy loss spectroscopy (EELS) for composition analysis. The TEM specimens were prepared by mechanically grinding small piece of material, and then amounting that on TEM Cu-grids.

Radioluminescence spectra were acquired using a ${ }^{90} \mathrm{Sr} /{ }^{90} \mathrm{Y}$ source (average beta energy $\sim 1 \mathrm{MeV}$ ), and were collected with a Princeton Instruments/Acton Spec 10 spectrograph coupled to a thermoelectrically cooled CCD camera and corrected for spectral sensitivity. Gamma ray spectra were acquired using a Hamamatsu R6231-100 superbialkali PMT. Plastic scintillators were centered on the entrance window, optically coupled to the PMT with optical grease and wrapped with several layers of Teflon tape. The PMT signals were shaped with a Tennelec TC 244 spectroscopy amplifier ( $8 \mu$ s shaping time for FIrpic activated samples and $1 \mu$ s for DPA activated samples) then recorded with an Amptek MCA8000-A multi-channel analyzer.

\section{DISCUSSION}

The discouraging results from pastattempts made to incorporate high- $Z$ dopants, for example $\mathrm{BiPh}_{3}$, into polymer scintillators in order to obtain a photopeak and enable gamma ray spectroscopy. ${ }^{1-3}$ were never fully explained in the literature, but can be understood if the relative excited state energies of the various materials are considered. Keeping with the examples of $\mathrm{BiPh}_{3}$ and PVT, the HOMO-LUMO gaps are $4.1 \mathrm{eV}^{5}$ and $4.2 \mathrm{eV}^{5}$ respectively. Energy transfer between the two is likely facile (fig 1a) and if the organometallic species either: (1) introduces a fast exciton relaxation pathway, or, (2) has a fast intersystem crossing to the triplet exciton state (a non-emissive state for the organic fluors in these systems), the light yield of the resulting scintillators will be significantly diminished.

These limitations in the $\mathrm{PVT} / \mathrm{BiPh}_{3}$ polymer scintillator system can be overcome with proper materials selection. The improved light yields reported by Campbell and Crone in the poly-vinyl carbazole (PVK)/Ir-dye scintillators suggest such materials. PVK has a smaller HOMO-LUMO gap $(3.5 \mathrm{eV})^{6}$ than $\mathrm{BiPh}_{3}$ and energy transfer from the organometallic dopant to the matrix is expected to be competitive with relaxation of the excited state and energy back transfer to the Bi dopant should not occur. Use of a high quantum yield phosphorescent dye should also improve the brightness of the scintillator while solving the possible problem of increased intersystem crossing induced by the heavy metal dopant. Care must be taken with the 
selection of the Ir dye however as the photomultiplier tubes used in gamma ray spectroscopy are maximally sensitive in the blue, and their sensitivity decreases beyond $500 \mathrm{~nm}$. FIrpic (fig 1c) was chosen as it was the shortest wavelength Ir dye commercially available when we began this study.
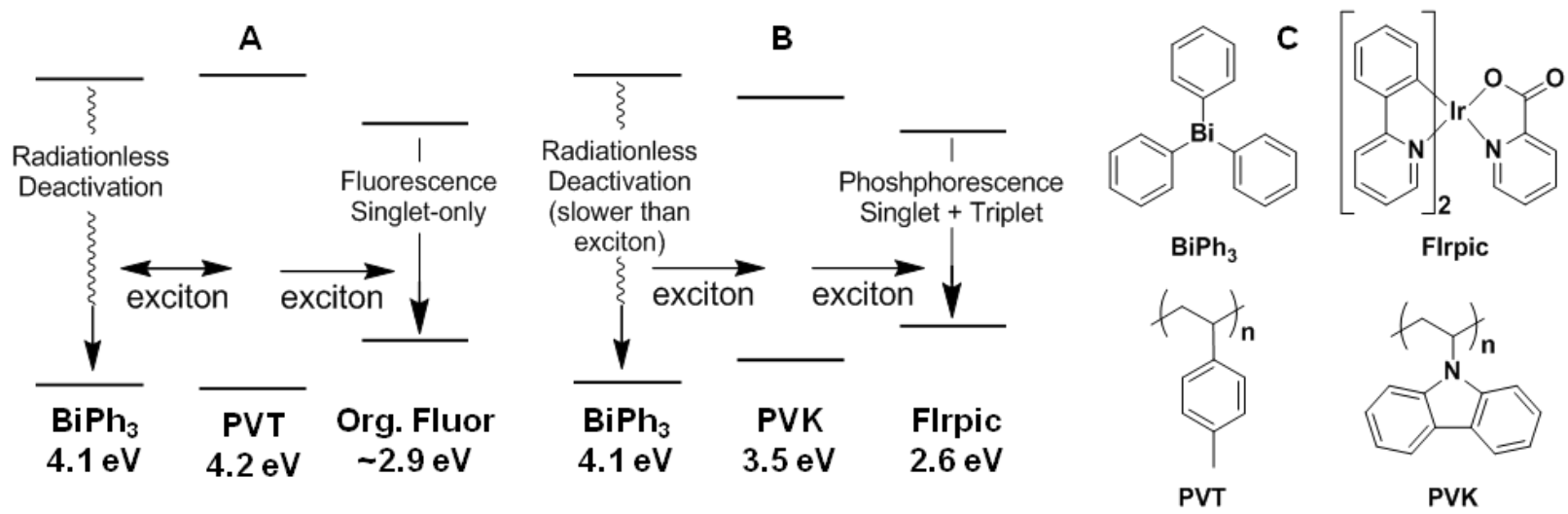

Figure 1. (A) Energy levels of Triphenyl Bismuth $\left(\mathrm{BiPh}_{3}\right)$, Polyvinyltoluene (PVT) and a typical organic fluor, Diphenyl Anthracene (DPA). (B) Energy levels of $\mathrm{BiPh}_{3}$, Polyvinylcarbazole (PVK) and an Iridium complex fluor (FIrpic). (C) Chemical structures of the constituent materials used in the high-Z polymer scintillators described in this work.

Table 1. Composition and summary of spectroscopy results of the samples used in this study.

\begin{tabular}{|c|c|c|c|c|c|c|}
\hline Sample & Matrix & Fluor & $\begin{array}{l}\mathrm{BiPh}_{3} \\
\text { Wt. \% }\end{array}$ & $\begin{array}{l}\beta \mathrm{LYY} \\
(\mathrm{Ph} / \mathrm{MeV})\end{array}$ & $\begin{array}{l}\text { Relative Gamma } \\
\text { Light Yield* }\end{array}$ & $\begin{array}{l}\text { Resolution } \\
\text { FWHM }^{*}\end{array}$ \\
\hline $1 \mathbf{a}$ & PVK & None & $0 \%$ & 1420 & $-¥$ & $-¥$ \\
\hline $1 b$ & PVK & None & $25 \%$ & _\$ & $-¥$ & $-¥$ \\
\hline $1 \mathrm{c}$ & PVK & None & $40 \%$ & $-\$$ & $-¥$ & $-\neq$ \\
\hline $2 \mathbf{a}$ & PVK & $3 \%$ DPA & $0 \%$ & 10120 & $-\neq$ & $-¥$ \\
\hline $2 b$ & PVK & $3 \%$ DPA & $25 \%$ & 6979 & $-¥$ & $-¥$ \\
\hline $2 c$ & PVK & $3 \%$ DPA & $40 \%$ & 6281 & 0.66 & $9 \%$ \\
\hline $3 \mathbf{a}$ & PVK & $1 \%$ FIrpic & $0 \%$ & 17400 & $-¥$ & $-\ldots$ \\
\hline $3 b$ & PVK & $1 \%$ FIrpic & $25 \%$ & 22069 & $-¥$ & $-¥$ \\
\hline $3 \mathbf{c}$ & PVK & $1 \%$ FIrpic & $40 \%$ & 25464 & $-¥$ & $-\ldots$ \\
\hline $4 \mathbf{a}$ & PVK & $3 \%$ FIrpic & $0 \%$ & 24191 & 0.73 & $9 \% *$ \\
\hline $4 b$ & PVK & $3 \%$ FIrpic & $25 \%$ & 25464 & $-\ldots$ & $-¥$ \\
\hline $4 c$ & PVK & $3 \%$ FIrpic & $40 \%$ & 30641 & 0.78 & $6.8 \%$ \\
\hline Eljen EJ208 & PVT & Proprietary & $0 \%$ & 17000 & 1.0 & $8 \% *$ \\
\hline
\end{tabular}

At $662 \mathrm{keV} \quad * *$ From fit to Compton edge ${ }^{7}$ Not Measured ${ }^{\text {s}}$ Value too small to measure

With these concepts in mind we made a series of twelve scintillator samples (Table 1) containing $40 \mathrm{wt} \%, 25 \mathrm{wt} \%$ or no $\mathrm{BiPh}_{3}$ and either no fluor, $3 \mathrm{wt} \%$ diphenyl anthracene (DPA) or $1 \mathrm{wt} \%$ or $3 \mathrm{wt} \%$ FIrpic. By examining this series we can separate out the effects of weight percent of Bi dopant, as well as the type and concentration of fluor (triplet and singlet harvesting 
in the case of FIrpic, or singlet-only fluorescence from DPA). All samples were compared to a commercial scintillator purchased from Eljen (EJ208, polyvinyl toluene based with singlet organic fluors) of comparable volume and shape. In producing these samples we found that processing parameters were extremely important. For example, recrystallization of NVK from methanol produced samples with a slight brown color, while sublimation produced lighter color and higher light yield samples. Temperature and radical initiator were also important variables. NVK melts around $65^{\circ} \mathrm{C}$ and this was the lowest temperature explored. This low temperature gave cloudy parts while heating to $80^{\circ} \mathrm{C}$ gave transparent parts with all components well dispersed in the sample. Heating above $80^{\circ} \mathrm{C}$ was problematic as a brown color which is assumed to be decomposition of thermally unstable $\mathrm{BiPh}_{3}$. At the chosen processing temperature many of the most common radical initiators have non-ideal decomposition kinetics. For example, AIBN decomposes too quickly at this temperature, resulting in a poorly controlled polymerization and bubbles in the solidified matrix. In contrast, many common peroxides such as tert-butyl peroxide decompose slowly and polymerization is often not complete for days. Luperox 231 allowed polyermization to be completed in 24 hours while still giving mechanically robust and bubble-free samples.

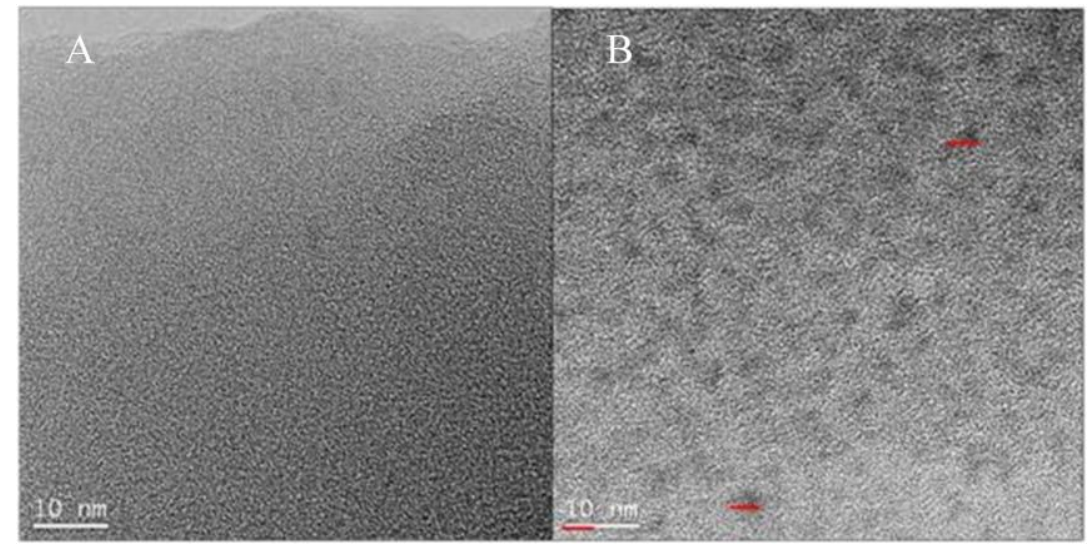

Figure 2. High resolution TEM of (A) Sample 3a and (B) 3c. White scale bars are $10 \mathrm{~nm}$ and red bars are $5 \mathrm{~nm}$.

Before beginning scintillation studies with these samples, we examined some by transmission electron microscopy (TEM) as high resolution phase contrast images can give us information about the dispersion state of $\mathrm{BiPh}_{3}$. TEM phase contrast images are presented in figure 2. Fig $2 \mathrm{a}$, which is an image of sample $\mathbf{3 a}\left(\right.$ no $\left.\mathrm{BiPh}_{3}\right)$ shows no obvious phase-separation, where as in sample 3c, $\left(40 \mathrm{wt} \% \mathrm{BiPh}_{3}\right)$ we find high-Z clusters even dispersed throughout the samples with diameter of about $5 \mathrm{~nm}$ (fig 2b). Samples with which contain $\mathrm{BiPh}_{3}$ at lower loadings (not shown) also showed high- $Z$ clusters of the same diameter. While this is larger than a single $\mathrm{BiPh}_{3}$ molecule, it likely represents a cluster of only several molecules. Attempts to copolymerize the Bi-dopant by using a vinyl derivative in either PVK or PVT resulted in similar clustering (not shown).

We began radioluminescence studies by making beta light yield ( $\beta \mathrm{LY}$ ) measurements on these samples. In this experiment, the samples exposed to high energy electrons, similar to the photoelectron ejected from a core orbital of a high-Z element during gamma spectroscopy, and the luminescence spectra are recorded. A spectrum is obtained, rather than counts with a photomultiplier tube (PMT), and is sensitive to the full range of wavelengths emitted by all of the dyes studied. This allows the samples to be checked for luminescence intensity without 
regard to spectral match to the PMT or to photoelectron efficiency as would be the case in gamma spectroscopy measurements. The first samples measured, 1a-c, have low light intensity, as expected, as they have no fluor and the quantum fluorescence quantum yield of PVK is low. In both the 25 and $40 \mathrm{wt} \% \mathrm{BiPh}_{3}$ samples light yields were below the detection limit. This indicates that there is still some quenching by the Bi-dopant from either a fast relaxation pathway or intersystem crossing to a triplet state. In samples $\mathbf{2 a - c}$ with DPA and varying $\mathrm{BiPh}_{3}$ loading, while light yields are substantially improved over the corresponding series 1a-c., the light yields still fall with increasing $\mathrm{BiPh}_{3}$ concentration.

All samples in series 3a-c and 4a-c are brighter in $\beta$ LY measurements than EJ208 and the brightness vs. $\mathrm{BiPh}_{3}$ loading trends are different. Each sample in series 4, with $3 \mathrm{wt} \%$ FIrpic, is brighter than the corresponding sample in series 3 with only $1 \mathrm{wt} \%$ Ir dye, albeit by a factor of less than $2 x$, indicating that not all excitons are being collected by the fluor in the lower concentration series. Higher concentrations of FIrpic have not yet been tested for higher beta light yields. Within each series $\mathbf{3}$ and $\mathbf{4}$, and in contrast to series $\mathbf{1}$ and $\mathbf{2}$, higher light yields are observed with increasing $\mathrm{BiPh}_{3}$ concentrations. Although the mechanism behind this phenomenon is not yet known, all observations so far are consistent with the hypothesis that the higher $\mathrm{BiPh}_{3}$ concentrations are resulting in a larger percentage of triplets in the system. This would reduce the light yield in the singlet-only emitters but might increase the light yields of triplet emitters if the triplet excitons were either more mobile or longer lived. Time resolved spectroscopy studies will hopefully allow a full mechanism to be elucidated in the future.

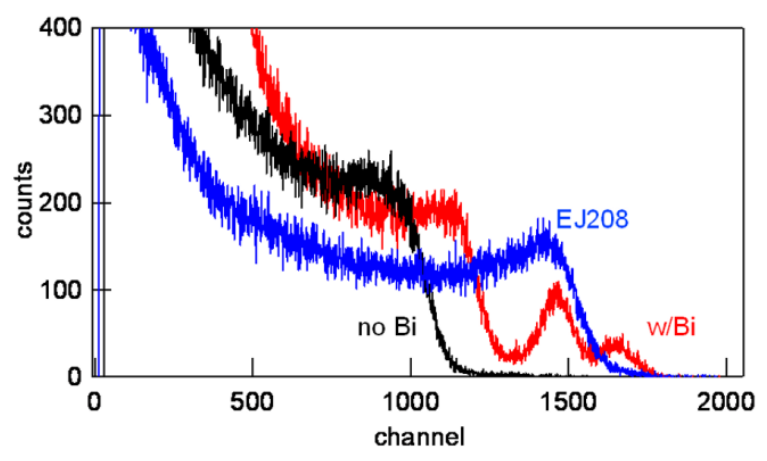

Figure 3. Cs-137 pulse height spectra of EJ208, 4a and 4c.

A subset of the samples were chosen for gamma ray spectroscopy using a Cs-137 source (662 keV gamma energy). Samples 2c, 4a, 4c and EJ208 were compared in order to determine the effect of Bi loading and the use of singlet vs. spin-orbit coupling fluor (4a, $\mathbf{4 c}$ and EJ208 shown in fig 3). Besides looking for a photopeak we also determined gamma light yields by direct comparison of the Compton edge with that of the EJ208 commercial sample, which was taken to have a light yield of 1 . The DPA sample $2 \mathrm{c}\left(40 \mathrm{wt} \% \mathrm{BiPh}_{3}\right)$, which had a significantly lower BLY than than EJ208, has a gamma light yield of 0.66. The samples $4 \mathbf{a}$ and $\mathbf{4 c}$, with the spin-orbit coupling fluor and 0 and $40 \mathrm{wt} \% \mathrm{BiPh}_{3}$, respectively, both had higher $\beta \mathrm{LY}$ than EJ208, but have relative gamma light yields of 0.73 and 0.78 . This lower light yield can be attributed to the spectral mismatch between the fluor and the PMT response. The increased light yield with added bismuth dopant in this case agrees with the $\beta$ LY observation.

The Cs-137 pulse height spectra reveal that the two $\mathrm{BiPh}_{3}$ doped samples, 2c and $\mathbf{4 c}$, have a photopeak. Sample $\mathbf{2 c}$ has a resolution of $9 \%$ while the higher light yield $\mathbf{4 c}$ has a resolution of $6.8 \%$ at $662 \mathrm{keV}$. Sample 4c's resolution is close to that of NaI (6\%), which is a 
commonly used scintillator material. In both of these samples two peaks are present, a full energy peak and an escape peak corresponding to the loss of a $77 \mathrm{keV}$ x-ray generated when an electron falls in to the vacancy in the core Bi shell created by the photo electron. This escape peak is greater in area than the full energy peak due to the moderate $\mathrm{Z}_{\text {eff }}$ of the samples and their small volume. In samples of sufficient volume, without any change in sample formulation, only the full energy peak should be present. The two samples lacking $\mathrm{BiPh}_{3}$ do not have a photopeak, clearly demonstrating the need for the high-Z dopant for spectroscopy applications.

\section{CONCLUSIONS}

In summary, polyvinylcarbazole-based plastic scintillators loaded with up to $40 \%$ triphenyl bismuth and activated with standard singlet fluors as well as spin-orbit coupling fluors may be formed with excellent mechanical integrity and high transparency. The light yields obtained with the Iridium complex spin-orbit coupling fluor (FIrpic) is about 3x that of the standard fluor, and the energy resolution with this sample is superior, as well. Energy resolution measured for the FIrpic-activated sample $662 \mathrm{keV}$ is $6.8 \%$, close to that of NaI. These initial results acquired with small samples are very promising, and scaleup to larger volumes is in progress. Large polymer scintillators offering equivalent performance to those reported here would have a wide range of uses, potentially replacing single crystals for some applications, and expanding the capabilities of plastic scintillators in applications where they are currently employed.

\section{ACKNOWLEDGMENTS}

This work was supported by the National Nuclear Security Administration, Office of Defense Nuclear Nonproliferation, Office of Nonproliferation Research and Development (NA22) of the U.S. Department of Energy under Contract No. DE-AC03-76SF00098, and performed under the auspices of the U.S. Department of Energy by Lawrence Livermore National Laboratory under Contract DE-AC52-07NA27344.

\section{REFERENCES}

1. 1. S. R. Sandler and K. C. Tsou, Int. J. Appl. Radiat. Is. 15, 419 (1963).

2. K. C. Tsou, IEEE T. Nucl. Sci., 28 (1965).

3. J. Dannin, S. R. Sandler and B. Baum, Int. J. Appl. Radiat. Is. 16, 589 (1965).

4. I. H. Campbell and B. K. Crone, Appl. Phys. Lett. 90, 012117 (2007).

5. Estimated from UV-vis.

6. F. J. Zhang, Z. Xu, D. W. Zhao, S. L. Zhao, L. W. Wang and G. C. Yuan, Phys. Scr. 77, 055403 (2008). 